\title{
Top-Down Attentional Deficits in Macaques with Lesions of Lateral Prefrontal Cortex
}

\author{
Andrew F. Rossi, ${ }^{1}$ Narcisse P. Bichot, ${ }^{2}$ Robert Desimone, ${ }^{2,3}$ and Leslie G. Ungerleider ${ }^{1}$ \\ Laboratories of ${ }^{1}$ Brain and Cognition and ${ }^{2}$ Neuropsychology, National Institute of Mental Health, National Institutes of Health, Bethesda, Maryland 20892, \\ and ${ }^{3} \mathrm{McG}$ covern Institute for Brain Research, Massachusetts Institute of Technology, Cambridge, Massachusetts 02139
}

Brain imaging, electrical stimulation, and neurophysiological studies have all implicated the prefrontal cortex (PFC) in the top-down control of attention. Specifically, feedback from PFC has been proposed to bias activity in visual cortex in favor of attended stimuli over irrelevant distracters. To identify which attentional functions are critically dependent on PFC, we removed PFC unilaterally in combination with transection of the corpus callosum and anterior commissure in two macaques. In such a preparation, the ipsilesional hemisphere is deprived of top-down feedback from PFC to visual cortex, and the contralesional hemisphere can serve as an intact normal control. Monkeys were trained to fixate a central cue and discriminate the orientation of a colored target grating presented among colored distracter gratings in either the hemifield affected by the PFC lesion or the normal control hemifield. Locations of the targets and distracters were varied, and the color of the central cue specified the color of the target on each trial. The behavioral response was a bar release, and thus attentional impairments could be distinguished from impaired oculomotor control. When the cue was held constant for many trials, task performance in the affected hemifield was nearly normal. However, the monkeys were severely impaired when the cue was switched frequently across trials. The monkeys were unimpaired in a pop-out task with changing targets that did not require top-down attentional control. The PFC thus appears to play a critical role in the ability to flexibly reallocate attention on the basis of changing task demands.

Key words: switching; primate; bottom-up; pop-out; salience; split brain

\section{Introduction}

The prefrontal cortex (PFC) has long been thought to play an integral role in the control of cognitive processes. Early studies in monkeys (Ferrier, 1876; Bianchi, 1922) and humans (Luria, 1969) described the effects of frontal damage as a disruption of goal-directed behaviors. More recent human lesion studies suggest a broad range of impairments in "executive function" and, in particular, attention (Knight, 1984; Duncan, 1986; Shallice and Burgess, 1991; Passingham, 1993; Rueckert and Grafman, 1996). Likewise, recent brain imaging studies have revealed a distributed network of areas in frontal and parietal cortex that appear to be involved in the allocation of attention, including the frontal eye field (FEF), supplementary eye field, anterior cingulate cortex, midfrontal gyrus, intraparietal sulcus, and superior parietal lobule (for review, see Hopfinger et al., 2000; Kastner and Ungerleider, 2000; Corbetta and Shulman, 2002).

Neurophysiological studies have also shown that neuronal responses in parts of PFC are related to attention (for review, see

\footnotetext{
Received June 28, 2007; revised Aug. 8, 2007; accepted Aug. 29, 2007.

This work was supported by the National Institute of Mental Health Intramural Research Program and National Institutes of Health Grant R01EY017292 (R.D.). We thank Grant Pielli, Ben Harris, and Poppy Rotter for assistance with animal training, Dr. Peter De Weerd for providing the programs for implementing the staircase procedure, and Dan Morris for assistance in programming the cueing experiments and data analysis. We also thank Dr. Richard S Saunders for assistance with surgical procedures and MRIs.

Correspondence should be addressed to Dr. Andrew F. Rossi, Department of Psychology, Vanderbilt University, 111 21st Avenue South, Room 301, Nashville, TN 37203. E-mail: andrew.rossi@vanderbilt.edu. D0I:10.1523/JNEUROSCI.2939-07.2007

Copyright $\odot 2007$ Society for Neuroscience ～0270-6474/07/2711306-09\$15.00/0
}

Schall, 2002) and the control of eye movements (for review, see Schall, 1997) as well as a variety of high-level behavioral functions, such as working memory (Goldman-Rakic, 1987; Quintana et al., 1988; Fuster, 1995; Miller et al., 1996), response strategies (Genovesio et al., 2005), and rule learning (Asaad et al., 1998, 2000; Rainer et al., 1998; White and Wise, 1999), all of which suggest that PFC neurons might modulate responses in sensory areas according to attention and task demands (for review, see Miller and Cohen, 2001). Direct evidence for this idea was provided by Moore and Armstrong (2003), who found that visual responses in area V4 could be enhanced after brief stimulation of retinotopically corresponding sites within the FEF and that stimulation of noncorresponding FEF representations could suppress V4 responses.

Together, these observations suggest the general hypothesis that PFC is one of a network of areas involved in the top-down control of attention by means of descending feedback signals that bias sensory processing in favor of information that is behaviorally relevant (Miller and D'Esposito, 2005). Several theories of attention have proposed that sensory representations compete for neural resources (Bundesen, 1990; Desimone and Duncan, 1995; Bundesen et al., 2005). For example, in the biased competition model (Desimone and Duncan, 1995), competition among sensory representations are resolved either through top-down feedback from frontal and parietal areas or by bottom-up sensory properties such as relative stimulus strength.

Because PFC is thought to be one of several structures in a 
larger attentional network, a question remains as to which attentional functions are critically dependent on PFC. Three experiments are presented here in which we explore the role of PFC in visual attention. We first describe the effect of PFC lesions on switching top-down control in a cued attention task. We next explore whether PFC is necessary for target selection when the target is determined by bottom-up stimulus salience. Finally, we examine the role of PFC in selecting a target in the presence of potent distracters.

\section{Materials and Methods}

Subjects and lesions. Two adult male macaque monkeys weighing between 8 and $10 \mathrm{~kg}$ were used in these experiments. Lesion and implant surgeries and behavioral testing were conducted according to National Institutes of Health guidelines and were approved by the National Institute of Mental Health Institutional Animal Care and Use Committee. Implant surgeries involved the placement of a post to immobilize the head and a scleral eye coil to monitor eye movements (Robinson, 1963). After removal of a large bone flap, comparable unilateral lesions of the lateral surface of prefrontal cortex of the right hemisphere were made in the two monkeys by aspiration of the gray matter (see Fig. 1a). The depth of the arcuate sulcus formed the caudal border of the lesion, which extended rostrally to the frontal pole. The lesion extended dorsomedially to the midline and ventromedially to the orbital gyrus, thereby including the FEF (area 8), dorsolateral areas 9 and 46, and ventrolateral areas 45 and 12. Medial prefrontal cortex and orbital prefrontal cortex were completely spared. The extent of the prefrontal lesion was verified postsurgically by a reconstruction of the remaining cortical structures from coronal slices obtained with magnetic resonance imaging (MRI) (GE Healthcare, Chalfont St. Giles, Buckinghamshire, UK; 1.5 T; 1-mm-thick slices; $256 \times 256$ pixel resolution; $11 \mathrm{~cm}$ field of view). In addition to the prefrontal lesion, the anterior commissure and corpus callosum were transected. As a result, visual processing in only the left hemisphere was potentially modulated by feedback from PFC, and we could assess the behavioral effects of the lesion by comparing visual performance in contralesional and ipsilesional hemifields in fixating monkeys. A reconstruction of the lesion is illustrated in Figure 1.

Procedure. Monkeys were trained to fixate a central cue on a display monitor and discriminate the orientation of the target grating presented among distracters in the periphery. Monkeys responded by either releasing a bar for a vertical grating or holding for nonvertical gratings and received a juice reward for all correct responses. The stimulus array of target and distracters was presented in either the left or right visual hemifield at a retinal eccentricity of $4^{\circ}$. The orientation of the target grating was determined using a staircase procedure (Wetherill and Levitt, 1965) that adjusted the magnitude of the orientation difference (from vertical) based on the monkey's previous performance. The orientation difference of the target grating was increased by $20 \%$ (relative to vertical) on error trials and decreased by $20 \%$ after three consecutive correct responses. In this way, an orientation threshold was determined for detecting the difference between vertical and nonvertical gratings, with performance typically converging to a level of $\sim 84 \%$ correct performance. The probability of a vertical target on any trial was $50 \%$. The staircase procedure terminated after a maximum of 120 trials or after 14 reversal points in the staircase. The orientation threshold was defined as the average of all reversal points except the initial four reversals. As a result, each threshold was based on $\sim 100$ trials. Thresholds based on fewer than five reversal points were discarded. Trials were aborted if the monkey's center of gaze fell outside a $2 \times 2^{\circ}$ fixation window centered on the cue.

Behavioral tasks and stimulus presentation. Both monkeys were trained on three variations of the orientation discrimination task. In experiment 1 , monkeys were trained to fixate a central cue and discriminate the orientation of a colored target grating presented among two colored distracter gratings. The color of the central cue (red, green, or blue) identified the target on each trial (see Fig. 1d). The cue appeared when the monkey held a lever to initiate a trial and remained present throughout the trial. Stimuli were colored sinusoidal gratings ( $50 \%$ Michelson contrast) of low spatial frequency $(1.5 \mathrm{cpd})$, photometrically isoluminant, and each contained in a circular aperture subtending $1.25^{\circ}$. The three gratings were arranged in a vertical linear array centered on the horizontal meridian, at $4^{\circ}$ eccentricity, and located $1.75^{\circ}$ apart. The gratings were presented for $500 \mathrm{~ms}$ (or less if the monkey responded during the grating presentation). On each trial, the relative positions of the colored gratings were randomly assigned unless otherwise indicated. In addition, the two distracters always differed in color and orientation from each other, to prevent the target from being identified solely on the basis of a feature difference from the distracters. We tested each monkey on four conditions of cue repetition: $1,5,10$, and 100 trials. For trials when the cue changed, the new color was randomly chosen from the two distracter colors in the previous trial.

In experiment 2, the monkeys were trained to fixate centrally and discriminate the orientation of the odd grating as defined by color pop-out (see Fig. $4 a$ ). Grating stimuli were the same as described in experiment 1 . Two additional distracters were added in this experiment to increase the salience of the target. The relative positions of the target and distracters were randomly assigned on each trial. Possible target positions were limited to the three most central locations nearest the horizontal meridian, as in experiment 1 . However, because the target was limited to the central three locations, it was always bordered by distracters on both sides, unlike most of the trials in experiment 1 . The identity of the target was systematically varied by changing either the color of the target, or both the color of the target and distracters. Similar to experiment 1, we tested each monkey on four conditions of target repetition: $1,5,10$, and 100 trials.

In experiment 3 , the target stimuli were phase-randomized achromatic gratings of low spatial frequency, presented in a circular aperture. The edges in the grating stimulus consisted of a $0.1^{\circ}$-wide random noise band having $50 \%$ pixels randomly turned on or off, which masked artifacts associated with the presentation of orientations close to principal axes of the cathode ray tube. Gratings were 50\% Michelson contrast, $1.5 \mathrm{cpd}$, presented in a circular aperture subtending $1.25^{\circ}$. The three distracters were uniformly bright disks of the same diameter as the target grating. The distracters were displayed in triplets surrounding the gratings (see Fig. $5 a$ ), and the configuration was random on each trial from eight predefined distracter configurations. The target grating position remained fixed in the lower quadrant of either the left or right hemifield. We systematically varied distracter contrast, relative to the background. Values of distracter contrast were $0,8,16,32$, and $64 \%$. Background luminance and the average grating luminance were equal and in the mesopic range.

Because both monkeys exhibited a bias for performing these tasks in the control hemifield, we often restricted testing to a single hemifield on a given day. This prevented the monkey from adopting a strategy in which stimuli presented in the lesion-affected hemifield were afforded little or no effort in anticipation of earning rewards with less effort when stimuli were presented in the control hemifield during the same testing session. A typical testing session resulted in the completion of 10-30 staircases $(\sim 1200-3600$ trials). During a given single session, one cue repetition condition was tested. The order of experimental conditions (e.g., cue repetition) was randomized across days. The data were analyzed using ANOVA and post hoc $t$ tests.

Both monkeys were trained on the basics of the distracter task (experiment 3 ) before the lesion, but without head fixation. Because the monkeys could simply fixate the target, the attentional requirement was minimal, and the main purpose of this pretraining was to teach the monkeys to perform an orientation discrimination. Both monkeys required several weeks of recovery from the lesion and bone-flap surgery before they could be implanted with a head post and trained. Postoperative experiments were preceded by training to achieve stable thresholds. The testing of monkey M1 in experiments 3,1 , and 2 began at 21,82 , and 146 weeks postlesion, respectively, and the testing of monkey M2 began at 37, 57, and 157 weeks postlesion, respectively. Thus, both monkeys had substantial periods to recover from the lesions, and any transient unilateral neglect resulting from the lesion would have been missed. The stability of the behavioral effects found in experiment 1 were confirmed by comparing orientation thresholds between test sessions occurring $\sim 2$ years apart ( 28 months apart for M1 and 20 months apart for M2); in neither monkey was there a significant improvement in performance when the color cue changed every trial (repetition 1). Finally, neither 
monkey demonstrated a response bias for the color or position of the target within a hemifield.

\section{Results \\ Experiment 1: effects of top-down switching}

The goal of this experiment was to investigate the effects of lateral PFC lesions on a monkey's ability to switch top-down control in a cued attention task. To accomplish this, two monkeys were trained to fixate a central cue and covertly attend to and discriminate the orientation of a colored target grating presented among colored distracter gratings. The color of the central cue identified the target on each trial (Fig. 1c). The orientation threshold for the target served as a measure of both acuity in the discrimination task and the ability to attend to the target in the presence of distracters. In other words, the target orientation threshold was used as an indirect measure of the monkey's ability to select the target as defined by the cue.

The results are shown in Figure $2 a$, where the average orientation threshold is plotted as a function of cue repetition for each monkey. Performance in the control hemifield is shown in white, and in the hemifield affected by the lesion in black. For both monkeys, the average orientation threshold across all levels of cue repetition was significantly greater in the lesion hemifield compared with the control hemifield (M1, $F_{(1,561)}=687.179, p<0.001 ; \mathrm{M} 2, F_{(1,596)}=$ $150.710, p<0.001)$ when the target was presented with distracters. Both monkeys also showed a small elevation in orientation discrimination thresholds in the lesionaffected hemifield compared with the control hemifield when the target was presented without distracters (see "no distracters" condition in Fig. $2 a$ ), which was significant in M1 ( $p=0.002$, $t$ test), but not in M2 ( $p=$ $0.539, t$ test). However, the average magnitude of the threshold impairment was significantly larger with distracters compared with without distracters for all cue repetition values in M1 ( $p<0.005, t$ test) and for all but the longest cue repetition value (100) in M2 ( $p<0.001, t$ test), consistent with the idea that the lesion caused an attentional impairment.

Although the lesion appeared to cause an attentional impairment, the magnitude of this impairment was strongly dependent on the rate of cue repetition. The threshold difference between the lesion and control hemifield was $28.7^{\circ}$ (M1) and $23.2^{\circ}$ (M2) when the cue changed every trial (repetition 1 ); however, this threshold difference dropped to $7.6^{\circ}(\mathrm{M} 1)$ and $1.1^{\circ}(\mathrm{M} 2)$ when the same cue was repeated for 100 trials. Both monkeys exhibited a systematic and significant increase in discrimination thresholds

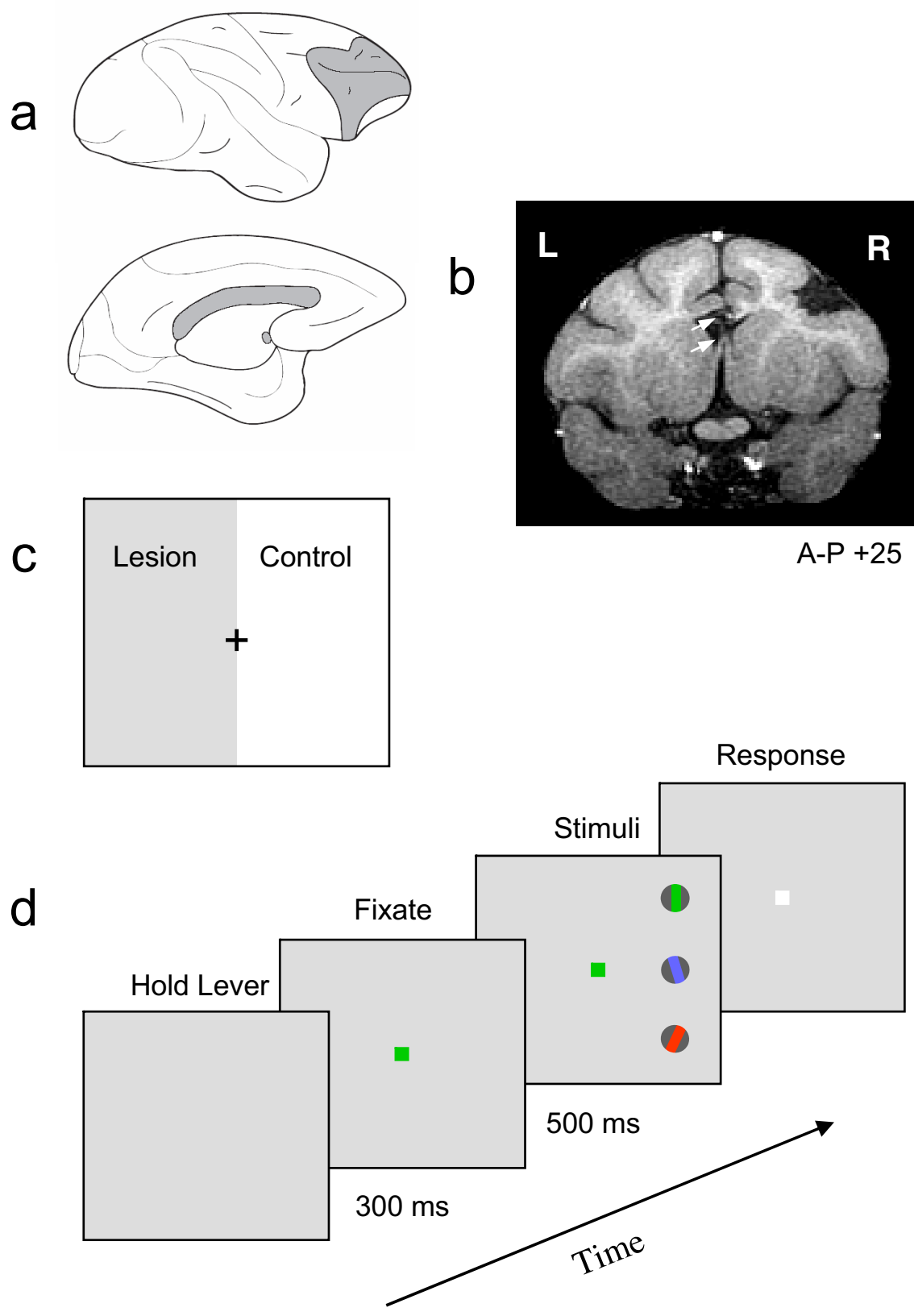

Figure 1. Methods and procedure. $\boldsymbol{a}$, Combination of unilateral PFC lesion and split brain. Top, The lateral view of the right hemisphere showing a lesion of the lateral surface of the right PFC (gray shading). Bottom, The medial surface of the left hemisphere shows the transection, in gray, of the corpus callosum and anterior commissure. $\boldsymbol{b}$, A postsurgical coronal section obtained with MRI from monkey M1 (for details, see Materials and Methods). This section, $25 \mathrm{~mm}$ anterior to the interaural stereotaxic landmark, shows a lesion of right lateral PFC defined medially by the superior arcuate sulcus and laterally by the inferior arcuate sulcus. In addition, the transection of the corpus callosum and anterior commissure is indicated by the top and bottom arrows, respectively. c, The combined lesion and split brain resulted in the contralesional visual hemifield, shown as gray, being processed without PFC and the ipsilesional visual hemifield serving as an experimental control. The effect of the lesion was assessed by comparing visual performance in the two hemifields. $\boldsymbol{d}$, The temporal sequence of stimulus presentation in the color cueing task. The monkey fixated centrally and discriminated the orientation of the peripheral grating that was cued by the color of the fixation spot. The relative positions of the colored gratings were randomly assigned each trial. The frequency at which the color cue changed was varied to examine the effect of increasing or decreasing the "top- down load" of the task. as the frequency of the cue change increased in the lesion hemifield $\left(\mathrm{M} 1, F_{(3)}=25.013, p<0.001 ; \mathrm{M} 2, F_{(3)}=20.591, p<0.001\right)$ but not in the control hemifield (M1, $p>0.1 ; \mathrm{M} 2, p>0.6)$, and there was a significant interaction between hemifield and cue repetition for both monkeys $\left(\mathrm{M} 1, F_{(3,561)}=53.523, p<0.001\right.$; 
a

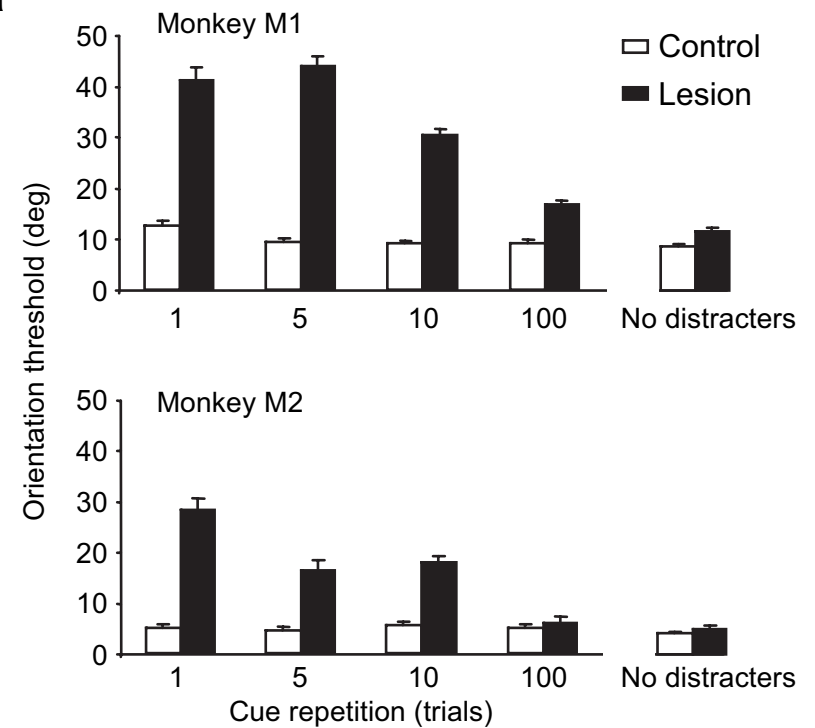

b

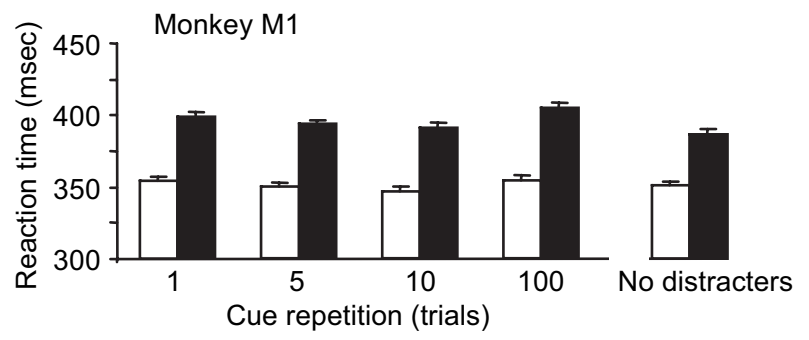

Figure 2. Effects of cue repetition on grating orientation discrimination in a color cueing task. $\boldsymbol{a}$, The average orientation threshold is plotted as a function of cue repetition for each monkey. Performance in the control hemifield is shown in white, and in the lesion-affected hemifield in black. Error bars represent the SEM. Each bar represents the average of between 40 and 60 thresholds for that condition. $\boldsymbol{b}$, The average reaction times for release trials as a function of cue repetition for monkey M1. Monkey M2 showed the same pattern of results.

$\left.\mathrm{M} 2, F_{(3,596)}=20.071, p<0.001\right)$. With 100 repetitions of the cue, the maximum tested, there was no longer a significant effect of the lesion in M2 $(p=0.617, t$ test $)$, but there was a small $\left(7.6^{\circ}\right.$ threshold elevation), significant impairment in monkey M1 ( $p=$ $0.014, t$ test). In sum, whereas the PFC lesion caused a small impairment in top-down attentional selection in one monkey when the target identify remained relatively fixed over trials, the major effect of the PFC lesion appeared to be an impairment in flexibly switching attention when the target identity changed frequently across trials.

\section{Reaction times}

In addition to the discrimination performance deficits described above, reaction times to stimuli presented in the lesion hemifield were relatively longer than in the control hemifield. Figure $2 b$ shows the average reaction time as a function of cue repetition for monkey $\mathrm{M} 1$ in the color cueing task. The average reaction time in the lesion hemifield was $\sim 400 \mathrm{~ms}$, compared with $350 \mathrm{~ms}$ in the control hemifield of this monkey. However, as illustrated in this figure, reaction time was fairly constant regardless of cue repetition. Monkey M2 (data not shown) showed a similar increase in reaction time in the lesion hemifield and the same independence of reaction time and cue repetition.

Effect of target location uncertainty

As described in Materials and Methods, the relative locations of the target and distracters were randomly chosen on each trial,

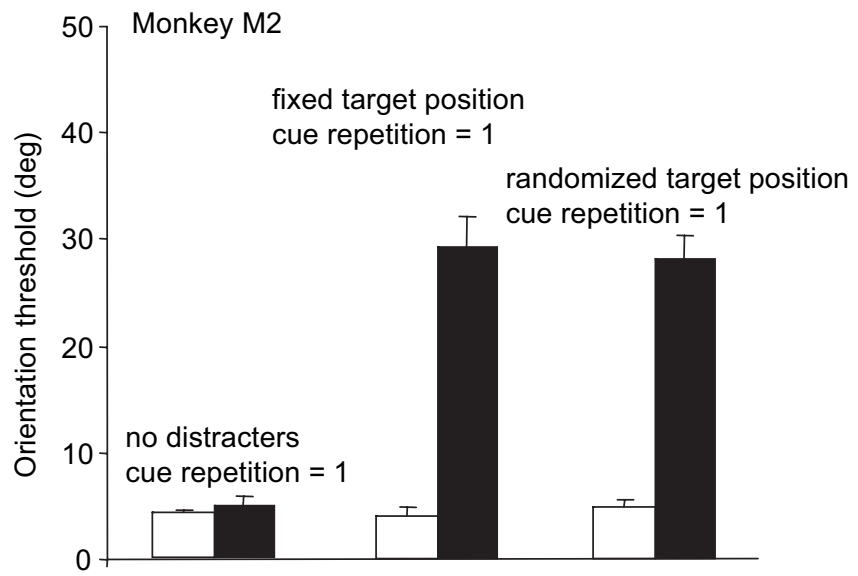

Figure 3. A comparison of three task conditions to explore the effects of target positional uncertainty on performance of the color cueing task. The bar graphs represent performance thresholds obtained with no distracters (left), randomly assigned target position (right; same as experiment 1), and fixed target position (middle). The black and white bars correspond to performance of the task in the contralesional and ipsilesional hemifields, respectively. Data shown are for M1. Error bars represent the SEM.

even when the cue was held constant for 100 trials. Thus, the impairment caused by increasing the cue switch frequency must have been attributable to the switch in cue or target identity (i.e., color) rather than any switch in target location across trials. Nonetheless, we considered the possibility that the impairment was caused not by an inability to direct attention to the appropriately colored target but rather by an inability to find (or spatially search for) a given target color among all the colored grating locations, when the target frequently switched color. If so, then reducing the spatial uncertainty of the target location by holding the locations of each of the stimulus colors fixed across trials should reduce the impairment. We therefore tested the performance of monkey M2 when the cue changed every trial and the colored grating locations were randomized on every trial, compared with when the location of each of the colored gratings remained fixed across trials (e.g., the red grating always in the upper location, the green grating always in the middle location, etc). We compared performance under these conditions to performance for the condition in which the target was randomly assigned to one of the three locations, but no distracters were present (same data as in Fig. 2a). As shown in Figure 3, the monkey had a large elevation in threshold regardless of whether the colored grating locations were fixed or varied across trials, suggesting that the impairment was not related to any spatial uncertainty in the colored grating locations.

\section{Analysis of errors}

If the PFC lesion impaired the ability of the monkeys to switch their attention to the new target color when the cue changed color over trials, then one would expect that a common error would be for the monkey to maintain attention on the relevant target color from the previous trial, even when the cue changed. That is, the monkeys might show a type of attentional perseveration for target features across trials.

To test this idea, we examined the pattern of errors across trials. This was not straightforward because of the covert nature of the attention task and the staircase design. Because the monkeys fixated centrally and did not move their eyes to the target, there was no definitive way to determine where the monkeys were 
attending during the trial. However, we were able to make inferences regarding the most likely types of errors that occurred.

We analyzed error trials based on a comparison of the type of response ("vertical" vs "nonvertical") on the error trial with the orientation of the two distracters on that trial. In the initial analysis, we assumed that the monkey made an error because it attended to, and discriminated, the orientation of a distracter rather than the target. We therefore analyzed each error trial that followed a correct trial by first determining whether the response was compatible with the orientation of one of the two distracters, and then examined whether that specific distracter shared the same color or the same location as the target used on the previous correct trial. In this manner, errors were classified as either "color error" or "location error," respectively. Error trials that could not be defined by these criteria were classified as "undefined."

Table 1 shows the outcome of this analysis for each monkey's performance in the contralesional hemifield. It can be seen that the majority of error trials could not be categorized (i.e., this method could not distinguish these trials as being solely consistent with the criterion for color or location perseveration). However, the percentage of errors that could be categorized increased with cue switch frequency, and the majority of these errors were consistent with a perseveration on the color of the previously rewarded target, but not its location. For both monkeys, the fraction of errors attributable to target color perseveration was significantly related to the frequency of change in the cue $\left(p<0.001, \chi^{2}\right.$ test), providing additional support for this interpretation.

An increase in perseveration at high cue switch frequency would also help explain the differences in thresholds and overall number of errors at each cue switch frequency. Table 1 shows that both monkeys made significantly more errors as the cue switch frequency was increased $\left(\mathrm{M} 1, F_{(3)}=10.13, p<0.05 ; \mathrm{M} 2, F_{(3)}=\right.$ 34.122, $p<0.01$ ), consistent with the analysis of perseveration errors described above. In the staircase testing protocol that we used, the orientation of the target grating was increased by $20 \%$ (relative to vertical) on error trials and decreased by $20 \%$ after three consecutive correct responses (see Materials and Methods). Thus, with a greater number of errors attributable to perseveration at high cue switch frequency, this would indirectly lead to higher orientation thresholds with high cue switch frequencies, which is what we found. Although these findings are only suggestive, the pattern of errors is consistent with the idea that the PFC lesion impaired the animals' flexibility to switch top-down control as informed by the color of the cue.

\section{Experiment 2: effect of target salience}

We next considered whether the impairment in switching attention to a new target across trials was attributable to a problem in guiding top-down attention based on information derived from the cue, or whether the impairment would be found in any task in which the target identity changed over trials, even if the "topdown" attentional requirement were reduced. We therefore trained the monkeys to perform a variation of the task in which the target was defined by color pop-out (Fig. 4a) (i.e., the monkeys were rewarded for discriminating the orientation of the grating that differed in color from all of the other gratings on a given trial). Thus, the target was defined by "bottom-up" stimulus features rather than the top-down information about the cue used in the task. The identity of the target was systematically varied across trials by changing either the color of the target, or the color of both the target and distracters. Similar to experiment 1 , we tested each monkey on four conditions of target repetition: $1,5,10$, and 100 trials.

Average discrimination thresholds for both monkeys are shown in Figure $4 b$. The thresholds in the control hemifield were larger than in experiment 1, possibly because of the larger number of distracters. The target was also bounded on both sides by grating distracters on every trial in this task, unlike most of the trials in experiment 1 . Both monkeys' thresholds were significantly higher in the lesion hemifield compared with the control hemifield $\left(\mathrm{M} 1, F_{(1,454)}=96.372, p=0.001 ; \mathrm{M} 2, F_{(1,428)}=8.336\right.$, $p=0.004)$, although the magnitude of the threshold increase for the lowest repetition values ( 1 and 5 ) was significantly smaller than in the color-cueing task ( $p<0.0001, t$ test). Monkey M1 also showed a significant increase in discrimination thresholds in both hemifields as the frequency of the target color change increased $\left(F_{(3,454)}=14.209 ; p<0.001\right)$. However, in neither monkey was the impairment caused by the frequent target color changes larger in the lesion hemifield compared with the control hemifield (i.e., there was not a significant interaction between hemifield and cue repetition value) $\left(\mathrm{M} 1, F_{(3,454)}=0.235, p=\right.$ $\left.0.872 ; \mathrm{M} 2, F_{(3,596)}=0.847, p=0.469\right)$. Thus, whereas performance in the pop-out attentional task was moderately impaired, this impairment did not seem related to the frequent switching of bottom-up attention to different salient targets across trials.

\section{Experiment 3: effect of distracter salience}

Previous studies of monkeys with large extrastriate lesions [encompassing areas V4 and/or temporal-occipital area (TEO)] found a large impairment in the inability to discriminate the orientation of a target in the presence of salient distracters (De Weerd et al., 1999). In those studies, the monkeys were not impaired when the target was much more salient (higher contrast) than the distracters; the impairment only emerged as the salience of the distracters was increased relative to the target. One possible interpretation of those results is that the large extrastriate lesion resulted in a disconnection of all top-down attentional feedback to visual cortex. In such a case, monkeys might be able to discriminate only those stimuli that stand out on the basis of bottom-up stimulus salience. If PFC were the major source of this top-down attentional feedback, we would expect that the monkeys in the 
a

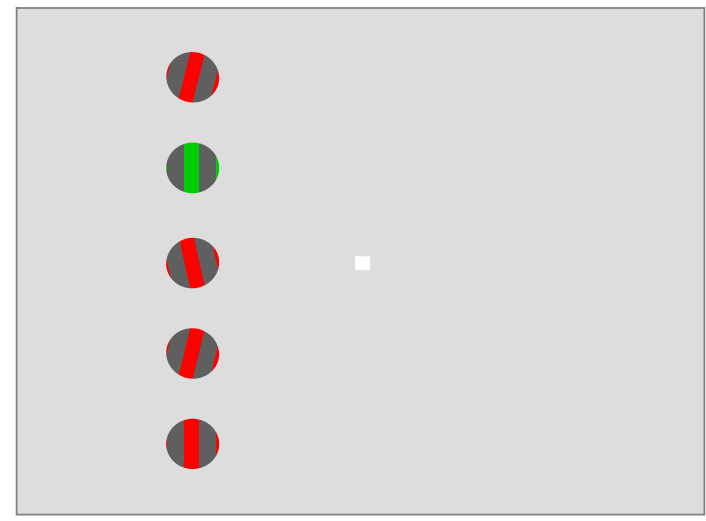

b

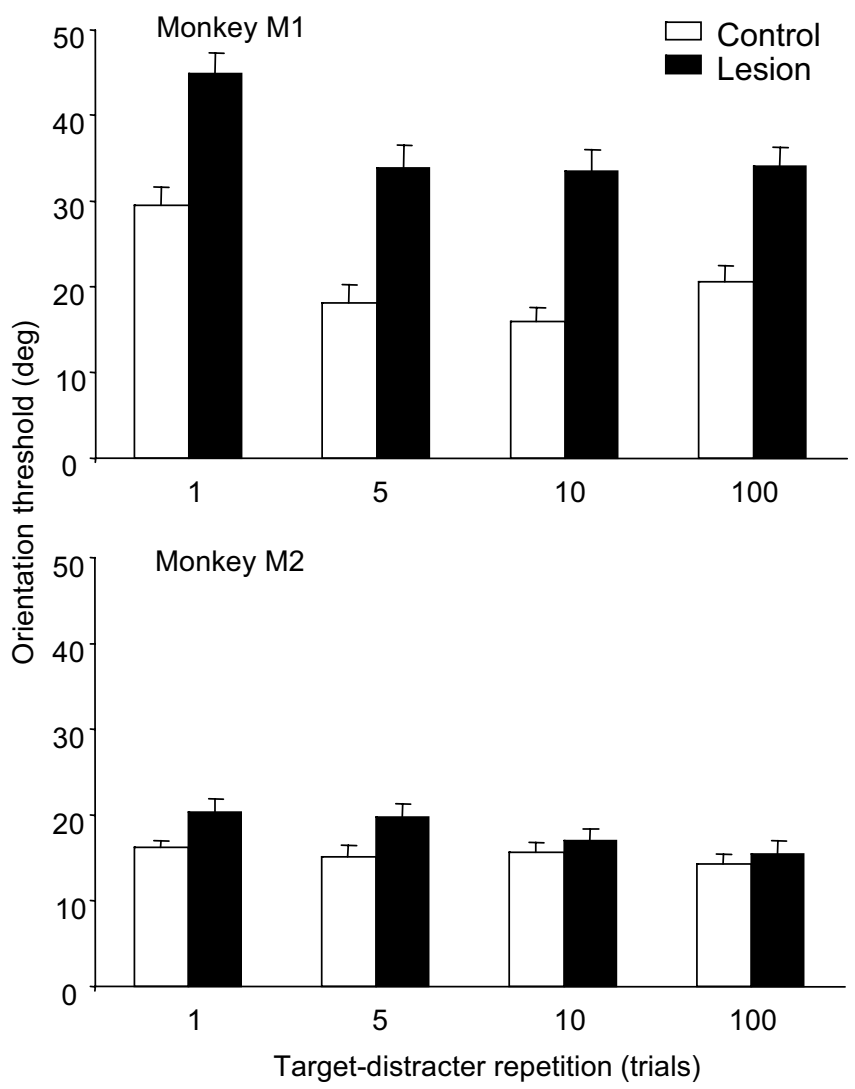

Figure 4. Effects of target-distracter repetition on grating orientation discrimination in a color pop-out task. $\boldsymbol{a}$, Color pop-out display. The temporal sequence of stimulus presentation was identical with that used in the color cueing task. The monkey fixated centrally and discriminated the orientation of the target grating as defined by color pop-out. The relative positions gratings were randomly assigned each trial. The frequency at which the target and distracter colors changed was varied to examine the effect of changes in target identity on task performance. $\boldsymbol{b}$, The average orientation threshold is plotted as function of cue repetition for each monkey (top and bottom plots). Performance in the control hemifield is shown in white, and in the lesion-affected hemifield in black. Error bars represent the SEM. Each bar represents the average of between 50 and 60 thresholds for that condition.

present experiment would show a similar impairment as the monkeys with V4 and TEO lesions.

Accordingly, we tested our monkeys on a task that was very similar to that used in De Weerd et al. (1999). In this task, monkeys were trained to discriminate the orientation of a single achromatic target grating surrounded by disk distracters of uniform a

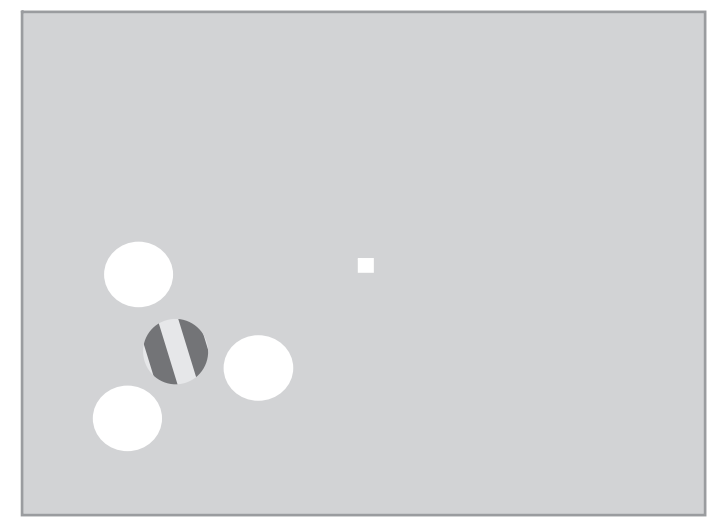

b

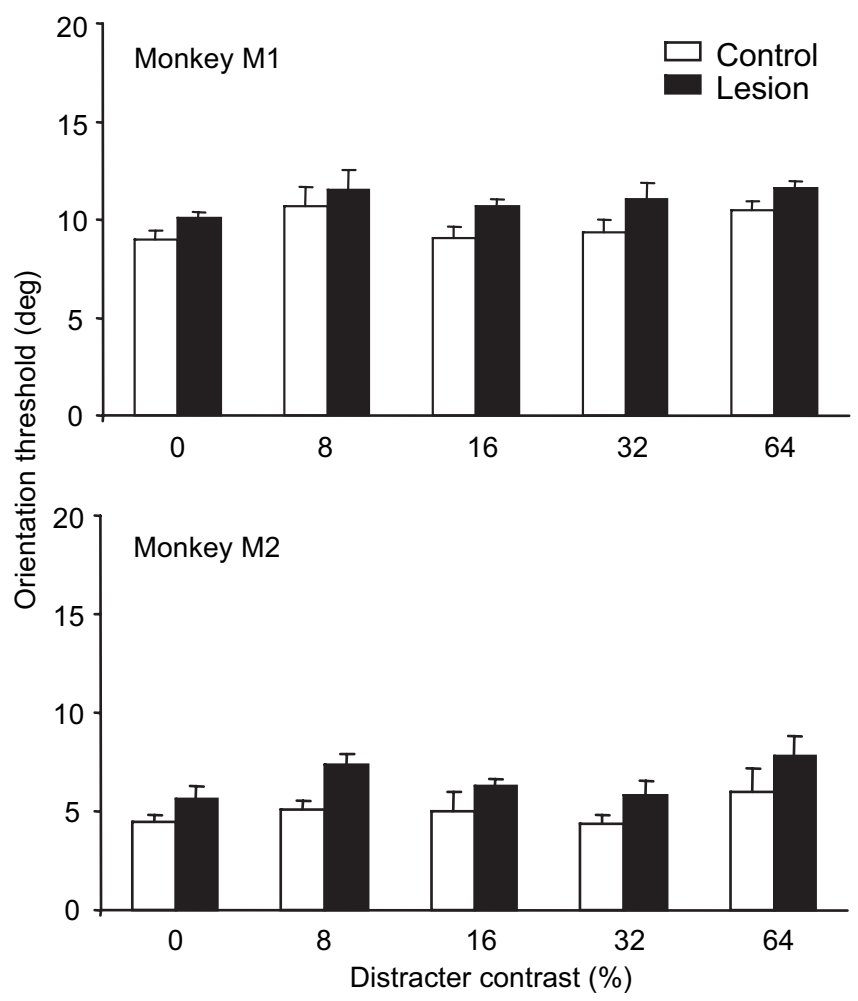

Figure 5. Effect of distracter contrast on grating orientation discrimination. $\boldsymbol{a}$, The target grating was surrounded by three disk distracters of uniform luminance. The monkey fixated centrally and discriminated the orientation of the target grating. The distracters were randomly assigned each trial. We systematically varied distracter contrast, relative to the background. Values of distracter contrast were 0 (i.e., no distracters), $8,16,32$, and $64 \%$. The target grating contrast was fixed at $50 \%$. $\boldsymbol{b}$, The average orientation threshold is plotted as function of distracter contrast for each monkey. Performance in the control hemifield is shown in white, and in the lesion-affected hemifield in black. Error bars represent the SEM. Each bar represents the average of between 40 and 50 thresholds for that condition.

luminance (Fig. 5a). The target identity remained constant over trials (always the only grating stimulus in the array) as did the relative location of the target compared with the distracters. However, the location of the entire array varied between the control and lesion hemifields. We tested five values of distracter contrast $(0,8,16,32$, and $64 \%$ Michelson contrast) to determine whether increasing distracter salience would have an effect on the monkeys' ability to attend to the target.

Both monkeys showed a small but significant threshold eleva- 
tion $\left(1.24^{\circ}\right.$ in $\mathrm{M} 1 ; 1.62^{\circ}$ in $\left.\mathrm{M} 2\right)$ in the lesion hemifield compared with the control $\left(\mathrm{M} 1, F_{(1,836)}=8.246, p=0.004 ; \mathrm{M} 2, F_{(1,427)}=\right.$ $17.512, p<0.001)$. However, there was no significant difference in average orientation threshold as a function of distracter contrast in either monkey $\left(\mathrm{M} 1, F_{(4,836)}=2.239, p=0.063 ; \mathrm{M} 2\right.$, $\left.F_{(4,427)}=1.876, p=0.114\right)$. Indeed, the impairment was the same when the distracters were invisible (Fig. $5 b, 0 \%$ contrast), in both monkeys. In other words, unlike what was found in the previous study with V4 and TEO lesions (De Weerd et al., 1999, 2003), the small but significant increase in orientation thresholds in the lesion hemifield did not depend on the magnitude of distracter contrast over the range of contrasts tested. Thus, consistent with the results from experiments 1 and 2, PFC does not appear to be critical in attending to a target stimulus if the monkeys are not required to switch their top-down selection of target features across trials.

\section{Discussion}

In experiment 1 , the animals' ability to use a central color cue to attend to a matching colored grating among distracters was impaired by PFC lesions, particularly when the cue/target changed frequently over trials. As the cue switch frequency increased, errors and the discrimination threshold for the target grating increased. Control experiments without distracters showed little or no impairment, arguing against a loss of acuity or a general inability of the monkey to attend.

Rather, the PFC lesion seemed to mainly impair the ability of monkeys to cognitively switch top-down attentional feedback from one target feature to another across trials (e.g., to attend to the red grating on one trial and the green grating on the next). An analysis of errors supports this interpretation, in that the monkeys seemed to perseverate in attending to the same target color from one trial to the next, even when the central cue indicated that they should attend to a different target color on that trial. Because the behavioral response was a bar release, we can rule out any switching impairment in oculomotor control or spatial orienting as a cause.

The results from the pop-out task in experiment 2 also support this interpretation of a top-down attentional switching impairment. We considered the pop-out task to be mainly a bottom-up task because the target was defined as the grating that differed in color from all of the others. Early studies concluded that detection of a pop-out stimulus occurs "preattentively," without requiring attention (Treisman and Gelade, 1980; Treisman and Souther, 1985) (for review, see Wolfe et al., 1998), whereas some subsequent studies argued that the recognition of pop-out targets does require attention, but that attention is drawn to them automatically (Theeuwes, 1994; Joseph and Optican, 1996; Joseph et al., 1997; Nothdurft, 1999). The most recent studies find that pop-out targets automatically attract attention only if the target is consistently defined to be the singleton stimulus in the display (Prinzmetal and Taylor, 2006). Thus, cognitive information about the task appears to modulate the processing of the pop-out stimulus. Performing an orientation discrimination, even on a target that automatically pops out from a display, clearly requires some type of attention; however, for the purposes of the present study, the critical feature of the pop-out task we used was that there was no information about target identity available to the animal at the start of the trial, and, thus, no top-down information about the target could be used to find it. We found that the animals did show a moderate impairment in this task, but it did not increase with the frequency of the target color change.
This result is consistent with a recent monkey study, comparing the role of PFC and parietal neurons in visual search (a topdown attention task) versus a pop-out task. In that study, PFC cells showed earlier attentional effects in the visual search task, whereas posterior parietal cells showed earlier attentional effects on the pop-out task (Buschman and Miller, 2007). Thus, although both areas appear to play some role in the performance of the pop-out task, the role of the parietal cortex may be predominant.

Experiment 3 was similar to experiment 2 in that the task did not require top-down information about target identity before the presentation of the stimulus array. Indeed, the target identity was constant throughout the experiment. The results were also comparable with those in experiment 2 , in that there was only a small increase in orientation threshold in the lesion hemifield. This impairment was constant as distracter contrast was increased, unlike the contrast-dependent distracter effects found previously after V4/TEO lesions (De Weerd et al., 1999, 2003). The dramatically different pattern of results after PFC versus V4/ TEO lesions suggests that PFC is not the only source of top-down attentional inputs to visual cortex. Rather, the pattern of results across all three experiments suggests that PFC is most needed for flexibly switching attention from one moment to the next, depending on which stimulus is relevant for the task at hand.

Numerous other studies have shown that unilateral lesions or reversible deactivation of the FEF and other parts of PFC often cause a transient contralateral neglect, which is typically most evident for orienting into the peripheral visual field or when there are competing stimuli in the unaffected parts of the visual field (extinction) (Welch and Stuteville, 1958; Crowne et al., 1981; Collin et al., 1982; Dias and Segraves, 1999; Iba and Sawaguchi, 2003; Schiller and Tehovnik, 2003; Wardak et al., 2006). Given that the neglect after a lesion typically resolves within a few days or weeks, and the deactivation effects last only hours, we could not observe such neglect in the present study because testing of the animals did not begin for several months after the lesions. The transient nature of the neglect suggests that PFC (including FEF) does contribute to attentional orienting in the intact animal but that these functions are shared by other components of a larger attentional network, as noted in Introduction. In addition to the PFC, imaging studies in humans typically show activation of one or more parietal regions as well as the thalamus and superior colliculus in attentional tasks (Gitelman et al., 2002) (for review, see Hopfinger et al., 2000; Kastner and Ungerleider, 2000; Corbetta and Shulman, 2002), and the lesions or deactivations of the same structures cause transient neglect and/or impaired oculomotor orienting in monkeys (Desimone et al., 1990; McPeek and Keller, 2004) (for review, see Robinson and Petersen, 1992; Schiller and Tehovnik, 2005). Consistent with this idea of shared function, combined lesions of PFC and either the parietal cortex (Lynch and McLaren, 1989; Lynch, 1992) or superior colliculus (Schiller et al., 1980) lead to much more severe, permanent effects on attentional orienting and eye movements than does dysfunction in either structure alone. However, the long-lasting impairments in attentional switching shown in the present study may reveal a critical function of the PFC, in much the same way that the permanent object discrimination impairments found after inferior temporal cortex lesions (for review, see Gross, 1973; Dean, 1982; Mishkin and Ungerleider, 1982) and memory impairments after medial temporal lobe lesions (for review, see Squire and Zola-Morgan, 1991; Squire et al., 2004) also reveal critical functions.

The idea that PFC plays a predominant role in the ability to 
flexibly switch top-down attention is compatible with human lesion data, which show that large lateral PFC lesions typically cause a long-lasting perseveration, either on a stimulus or on a response (Milner, 1963; Walker et al., 1998; Manes et al., 2002; Aron et al., 2004). As with our monkeys, these studies show that PFC lesions typically do not result in a permanent loss of the ability to attend selectively. Likewise, functional MRI studies in humans often show activation of PFC in attentional tasks, but the activation is greatest when subjects must switch between responses, switch between tasks, or switch their attention between different stimuli (Dove et al., 2000; Monchi et al., 2001; Dreher and Berman, 2002; Dreher et al., 2002; Brass et al., 2003; Braver et al., 2003; Dreher and Grafman, 2003; Smith et al., 2004; Hampshire and Owen, 2006; Loose et al., 2006; Slagter et al., 2006; Yeung et al., 2006). In monkeys, PFC cells switch their response properties between different tasks, and even between different phases of the same task (Hoshi et al., 1998; White and Wise, 1999; Asaad et al., 2000; Buschman and Miller, 2007). The present results support the idea that PFC lesions in monkeys, like those in humans, have long-lasting effects on attentional switching, which other brain structures apparently cannot fully compensate for over time (Mishkin, 1964; Knight, 1984; Dias et al., 1996a,b; Chao and Knight, 1997; Rushworth et al., 1997).

\section{References}

Aron AR, Monsell S, Sahakian BJ, Robbins TW (2004) A componential analysis of task-switching deficits associated with lesions of left and right frontal cortex. Brain 127:1561-1573.

Asaad WF, Rainer G, Miller EK (1998) Neural activity in the primate prefrontal cortex during associative learning. Neuron 21:1399-1407.

Asaad WF, Rainer G, Miller EK (2000) Task-specific neural activity in the primate prefrontal cortex. J Neurophysiol 84:451-459.

Bianchi L (1922) The mechanism of the brain and the function of the frontal lobes. Edinburgh: Livingstone.

Brass M, Ruge H, Meiran N, Rubin O, Koch I, Zysset S, Prinz W, von Cramon DY (2003) When the same response has different meanings: recoding the response meaning in the lateral prefrontal cortex. NeuroImage 20:1026-1031.

Braver TS, Reynolds JR, Donaldson DI (2003) Neural mechanisms of transient and sustained cognitive control during task switching. Neuron 39:713-726.

Bundesen C (1990) A theory of visual attention. Psychol Rev 97:523-547.

Bundesen C, Habekost T, Kyllingsbaek S (2005) A neural theory of visual attention: bridging cognition and neurophysiology. Psychol Rev 112:291-328.

Buschman TJ, Miller EK (2007) Top-down versus bottom-up control of attention in the prefrontal and posterior parietal cortices. Science 315:1860-1862.

Chao LL, Knight RT (1997) Prefrontal deficits in attention and inhibitory control with aging. Cereb Cortex 7:63-69.

Collin NG, Cowey A, Latto R, Marzi C (1982) The role of frontal eye-fields and superior colliculi in visual search and non-visual search in rhesus monkeys. Behav Brain Res 4:177-193.

Corbetta M, Shulman GL (2002) Control of goal-directed and stimulusdriven attention in the brain. Nat Rev Neurosci 3:201-215.

Crowne DP, Yeo CH, Russell IS (1981) The effects of unilateral frontal eye field lesions in the monkey: visual-motor guidance and avoidance behaviour. Behav Brain Res 2:165-187.

Dean P (1982) Visual behavior in monkeys with inferotemporal lesions. In: Analysis of visual behavior (Ingle DJ, Goodale MA, Mansfield RJW, eds), pp 587-628. Cambridge, MA: MIT.

Desimone R, Duncan J (1995) Neural mechanisms of selective visual attention. Annu Rev Neurosci 18:193-222.

Desimone R, Wessinger M, Thomas L, Schneider W (1990) Attentional control of visual perception: cortical and subcortical mechanisms. Cold Spring Harb Symp Quant Biol 55:963-971.

De Weerd P, Peralta III MR, Desimone R, Ungerleider LG (1999) Loss of attentional stimulus selection after extrastriate cortical lesions in macaques. Nat Neurosci 2:753-758.
De Weerd P, Desimone R, Ungerleider LG (2003) Impairments in spatia generalization of visual skills after V4 and TEO lesions in macaques ( $\mathrm{Ma}$ caca mulatta). Behav Neurosci 117:1441-1447.

Dias EC, Segraves MA (1999) Muscimol-induced inactivation of monkey frontal eye field: effects on visually and memory-guided saccades. J Neurophysiol 81:2191-2214.

Dias R, Robbins TW, Roberts AC (1996a) Dissociation in prefrontal cortex of affective and attentional shifts. Nature 380:69-72.

Dias R, Robbins TW, Roberts AC (1996b) Primate analogue of the Wisconsin Card Sorting Test: effects of excitotoxic lesions of the prefrontal cortex in the marmoset. Behav Neurosci 110:872-886.

Dove A, Pollmann S, Schubert T, Wiggins CJ, von Cramon DY (2000) Prefrontal cortex activation in task switching: an event-related fMRI study. Brain Res Cogn Brain Res 9:103-109.

Dreher JC, Berman KF (2002) Fractionating the neural substrate of cognitive control processes. Proc Natl Acad Sci USA 99:14595-14600.

Dreher JC, Grafman J (2003) Dissociating the roles of the rostral anterior cingulate and the lateral prefrontal cortices in performing two tasks simultaneously or successively. Cereb Cortex 13:329-339.

Dreher JC, Koechlin E, Ali SO, Grafman J (2002) The roles of timing and task order during task switching. NeuroImage 17:95-109.

Duncan J (1986) Disorganization of behaviour after frontal lobe damage. Cogn Neuropsychol 3:270-290.

Ferrier D (1876) The functions of the brain. New York: Putnam.

Fuster JM (1995) Memory in the cerebral cortex. Cambridge, MA: MIT.

Genovesio A, Brasted PJ, Mitz AR, Wise SP (2005) Prefrontal cortex activity related to abstract response strategies. Neuron 47:307-320.

Gitelman DR, Parrish TB, Friston KJ, Mesulam MM (2002) Functional anatomy of visual search: regional segregations within the frontal eye fields and effective connectivity of the superior colliculus. NeuroImage 15:970-982.

Goldman-Rakic P (1987) Circuitry of primate prefrontal cortex and regulation of behavior by representational memory. In: Handbook of physiology: the nervous system (Plum F, ed), pp 373-417. Bethesda, MD: Am Physiol Soc.

Gross CG (1973) Visual functions of inferotemporal cortex. In: Handbook of sensory physiology (Jung R, ed), pp 451-482. Berlin: Springer.

Hampshire A, Owen AM (2006) Fractionating attentional control using event-related fMRI. Cereb Cortex 16:1679-1689.

Hopfinger JB, Buonocore MH, Mangun GR (2000) The neural mechanisms of top-down attentional control. Nat Neurosci 3:284-291.

Hoshi E, Shima K, Tanji J (1998) Task-dependent selectivity of movementrelated neuronal activity in the primate prefrontal cortex. J Neurophysiol 80:3392-3397.

Iba M, Sawaguchi T (2003) Involvement of the dorsolateral prefrontal cortex of monkeys in visuospatial target selection. J Neurophysiol 89:587-599.

Joseph JS, Optican LM (1996) Involuntary attentional shifts due to orientation differences. Percept Psychophys 58:651-665.

Joseph JS, Chun MM, Nakayama K (1997) Attentional requirements in a "preattentive" feature search task. Nature 387:805-807.

Kastner S, Ungerleider LG (2000) Mechanisms of visual attention in the human cortex. Annu Rev Neurosci 23:315-341.

Knight RT (1984) Decreased response to novel stimuli after prefrontal lesions in man. Electroencephalogr Clin Neurophysiol 59:9-20.

Loose R, Kaufmann C, Tucha O, Auer DP, Lange KW (2006) Neural networks of response shifting: influence of task speed and stimulus material. Brain Res 1090:146-155.

Luria AR (1969) Frontal lobe syndromes. In: Handbook of clinical neurology (Vinken PJ, Bruyn GW, eds), pp 725-757. New York: Elsevier.

Lynch JC (1992) Saccade initiation and latency deficits after combined lesions of the frontal and posterior eye fields in monkeys. J Neurophysiol 68:1913-1916.

Lynch JC, McLaren JW (1989) Deficits of visual attention and saccadic eye movements after lesions of parietooccipital cortex in monkeys. J Neurophysiol 61:74-90

Manes F, Sahakian B, Clark L, Rogers R, Antoun N, Aitken M, Robbins T (2002) Decision-making processes following damage to the prefrontal cortex. Brain 125:624-639.

McPeek RM, Keller EL (2004) Deficits in saccade target selection after inactivation of superior colliculus. Nat Neurosci 7:757-763. 
Miller EK, Cohen JD (2001) An integrative theory of prefrontal cortex function. Annu Rev Neurosci 24:167-202.

Miller EK, Erickson CA, Desimone R (1996) Neural mechanisms of visual working memory in prefrontal cortex of the macaque. J Neurosci 16:5154-5167.

Miller LM, D'Esposito M (2005) Perceptual fusion and stimulus coincidence in the cross-modal integration of speech. J Neurosci 25:5884-5893.

Milner B (1963) Effects of different brain lesion on card sorting. Arch Neurol 9:90.

Mishkin M (1964) Perseveration of central sets after frontal lesions in monkeys. In: The frontal granular cortex and behavior (Warren JM, Alkert K, eds), pp 219-241. New York: McGraw-Hill.

Mishkin M, Ungerleider LG (1982) Contribution of striate inputs to the visuospatial functions of parieto-preoccipital cortex in monkeys. Behav Brain Res 6:57-77.

Monchi O, Petrides M, Petre V, Worsley K, Dagher A (2001) Wisconsin Card Sorting revisited: distinct neural circuits participating in different stages of the task identified by event-related functional magnetic resonance imaging. J Neurosci 21:7733-7741.

Moore T, Armstrong KM (2003) Selective gating of visual signals by microstimulation of frontal cortex. Nature 421:370-373.

Nothdurft HC (1999) Focal attention in visual search. Vision Res 39:2305-2310.

Passingham R (1993) The frontal lobes and voluntary action. Oxford: Oxford UP.

Prinzmetal W, Taylor N (2006) Color singleton pop-out does not always poop out: an alternative to visual search. Psychon Bull Rev 13:576-580.

Quintana J, Yajeya J, Fuster JM (1988) Prefrontal representation of stimulus attributes during delay tasks. I. Unit activity in cross-temporal integration of sensory and sensory-motor information. Brain Res 474:211-221.

Rainer G, Asaad WF, Miller EK (1998) Selective representation of relevant information by neurons in the primate prefrontal cortex. Nature 393:577-579.

Robinson DA (1963) A method of measuring eye movement using a scleral search coil in a magnetic field. IEEE Trans Biomed Eng 10:137-145.

Robinson DL, Petersen SE (1992) The pulvinar and visual salience. Trends Neurosci 15:127-132.

Rueckert L, Grafman J (1996) Sustained attention deficits in patients with right frontal lesions. Neuropsychologia 34:953-963.

Rushworth MF, Nixon PD, Eacott MJ, Passingham RE (1997) Ventral prefrontal cortex is not essential for working memory. J Neurosci 17:4829-4838.

Schall JD (1997) Visuomotor areas of the frontal lobe. In: Cerebral cortex (Rockland K, Kaas JH, Peters A, eds). New York: Plenum.
Schall JD (2002) The neural selection and control of saccades by the frontal eye field. Philos Trans R Soc Lond B Biol Sci 357:1073-1082.

Schiller PH, Tehovnik EJ (2003) Cortical inhibitory circuits in eyemovement generation. Eur J Neurosci 18:3127-3133.

Schiller PH, Tehovnik EJ (2005) Neural mechanisms underlying target selection with saccadic eye movements. Prog Brain Res 149:157-171.

Schiller PH, True SD, Conway JL (1980) Deficits in eye movements following frontal eye-field and superior colliculus ablations. J Neurophysio 44:1175-1189.

Shallice T, Burgess PW (1991) Deficits in strategy application following frontal lobe damage in man. Brain 114:727-741.

Slagter HA, Weissman DH, Giesbrecht B, Kenemans JL, Mangun GR, Kok A Woldorff MG (2006) Brain regions activated by endogenous preparatory set shifting as revealed by fMRI. Cogn Affect Behav Neurosci 6:175-189.

Smith AB, Taylor E, Brammer M, Rubia K (2004) Neural correlates of switching set as measured in fast, event-related functional magnetic resonance imaging. Hum Brain Mapp 21:247-256.

Squire LR, Zola-Morgan S (1991) The medial temporal lobe memory system. Science 253:1380-1386.

Squire LR, Stark CE, Clark RE (2004) The medial temporal lobe. Annu Rev Neurosci 27:279-306.

Theeuwes J (1994) Stimulus-driven capture and attentional set: selective search for color and visual abrupt onsets. J Exp Psychol Hum Percept Perform 20:799-806.

Treisman A, Souther J (1985) Search asymmetry: a diagnostic for preattentive processing of separable features. J Exp Psychol Gen 114:285-310.

Treisman AM, Gelade G (1980) A feature-integration theory of attention. Cognit Psychol 12:97-136.

Walker R, Husain M, Hodgson TL, Harrison J, Kennard C (1998) Saccadic eye movement and working memory deficits following damage to human prefrontal cortex. Neuropsychologia 36:1141-1159.

Wardak C, Ibos G, Duhamel JR, Olivier E (2006) Contribution of the monkey frontal eye field to covert visual attention. J Neurosci 26:4228 -4235.

Welch K, Stuteville P (1958) Experimental production of unilateral neglect in monkeys. Brain 81:341-347.

Wetherill GB, Levitt H (1965) Sequential estimation of points on a psychometric function. Br J Math Stat Psychol 18:1-10.

White IM, Wise SP (1999) Rule-dependent neuronal activity in the prefrontal cortex. Exp Brain Res 126:315-335.

Wolfe JM, O’Neill P, Bennett SC (1998) Why are there eccentricity effects in visual search? Visual and attentional hypotheses. Percept Psychophys 60:140-156.

Yeung N, Nystrom LE, Aronson JA, Cohen JD (2006) Between-task competition and cognitive control in task switching. J Neurosci 26:1429-1438. 